Discussion Paper No. 1007

\title{
DEMAND UNCERTAINTY, PRODUCT DIFFERENTIATION, AND ENTRY TIMING UNDER SPATIAL COMPETITION
}

\author{
Takeshi Ebina
}

Noriaki Matsushima

Katsumasa Nishide

July 2017

The Institute of Social and Economic Research Osaka University

6-1 Mihogaoka, Ibaraki, Osaka 567-0047, Japan 


\title{
Demand Uncertainty, Product Differentiation, and Entry Timing under Spatial Competition
}

\author{
Takeshi Ebina* \\ Faculty of Economics, Shinshu University \\ Noriaki Matsushima ${ }^{\dagger}$ \\ Institute of Social and Economic Research, Osaka University \\ Katsumasa Nishide \\ Graduate School of Economics, Hitotsubashi University
}

July 4, 2017

\begin{abstract}
We investigate the entry timing and location decisions under market-size uncertainty with Brownian motions in a continuous-time spatial competition duopoly model a la d'Aspremont et al. (1979). Under a sequential equilibrium, the threshold of the follower non-monotonically increases in volatility, which is in stark contrast to the extant results in the real options literature. Also, although the follower's entry timing tends to be late as the volatility becomes amplified, the leader is more likely to increase the degree of product differentiation as the volatility gets higher. Finally, we compare the equilibrium entry decisions with the second-best ones.
\end{abstract}

Keywords: Location; Hotelling model; Continuous-time model; Entry timing; Real options.

JEL classification: C73, D81, L11, L13,

${ }^{*}$ Faculty of Economics, Shinshu University, 3-1-1, Asahi, Matsumoto, Nagano, Japan. Phone: (81)-48021-7663. Fax: (81)-4-8021-7654. E-mail: ebina@shinshu-u.ac.jp

${ }^{\dagger}$ Institute of Social and Economic Research, Osaka University, Mihogaoka 6-1, Ibaraki, Osaka, Japan. Phone: (81)-6-6879-8571. Fax: (81)-6-6879-8583. E-mail: nmatsush@iser.osaka-u.ac.jp

${ }^{\ddagger}$ Graduate School of Economics, Hitotsubashi University, 2-1 Naka, Kunitachi, Tokyo, 186-8601, Japan. Phone: (81)-42-580-8856. FAX: (81)-42-580-8856. E-mail: k.nishide@r.hit-u.ac.jp 


\section{Introduction}

When a firm launches new products and/or services in a market, determining the product characteristics is one of its important decisions. For example, a fast food chain operator opening a new restaurant needs to decide its location considering the demand in the area as well as the competition with other firms. Another example is a manufacturing company that considers what kind of products to develop taking into account the future demand. In the marketing literature, several studies such as Bronnenberg and Mahajan (2001), and Cleeren et al. (2010) show that product positions greatly influence the pricing decisions of retailing and marketing firms.

Because of the importance of product positioning, many theoretical studies investigate the factors determining the product positions of firms in various contexts by using the Hotelling linear city models. These studies include d'Aspremont et al. (1979), Friedman and Thisse (1993), Tabuchi and Thisse (1995), Kim and Serfes (2006), Matsushima (2009), and Lai and Tabuchi (2012) @ Although the existing studies offer many interesting insights, they are derived using static or discrete-time models with only a few periods because consumers purchase products at most several times, implying robust results over the markets where the demand is quite stable for a long time. However, if we consider the consumers' repeated purchases in growing or changing markets, entry timing is an important strategic decision for firms.

In actual situations, the uncertainty of future demand of products affect the decisions on location, product positioning, entry timing, and so on. Uncertainty is especially important for firms facing competition because the strategies of entry timing and product positioning may have a bigger and mutual impact on the strategies of other firms without uncertainty. In summary, a firm needs to take uncertainty and competition into account

\footnotetext{
${ }^{1}$ Several empirical studies also investigate the problems of positioning (e.g., Thomadsen, 2007, Hwang et al., 2010).
} 
when deciding on product positioning and entry timing.2

Recently, Huisman and Kort (2015) studied the problem where two asymmetric firms choose not only their investment timing but also their production capacity at investment. Market size is a state variable and follows a geometric Brownian motion as in the real options literature ${ }^{3}$ Huisman and Kort (2015) is novel in that two symmetric firms optimally choose two strategic variables. This approach can be applied to many industrial

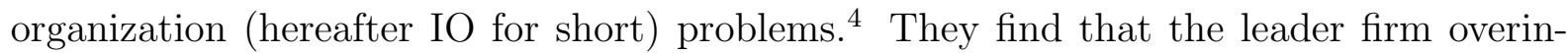
vests in capacity to deter the entry of the follower and that greater uncertainty makes entry deterrence more likely. From their article, uncertainty is of great importance for the decision making of firms facing competition, and that the multiple dimension of decision making may lead to different results compared to a single-dimension case.

In the IO literature, Ebina et al. (2015) extend Lambertini (2002) to study the entry timing of firms facing competition. They construct a continuous-time spatial competition model a la d'Aspremont et al. (1979). In the model, two firms optimally determine their locations and prices as well as the follower's entry timing, but the leader's entry time is exogenously fixed as in Lambertini (2002). The main finding of the study is that the leader has an incentive to locate close to the center to delay the follower's entry, leading to a non-maximum outcome from the viewpoint of social welfare. The study also sheds light on the importance of multiple dimensions for the firm's decision-making. It is noteworthy that the demand for the product grows at a constant rate, indicating no uncertainty in their model.

In this article, we investigate the entry decisions of firms that endogenously determine their product positions in a market whose size is evolving stochastically. To this end, we

\footnotetext{
${ }^{2}$ In the context of spatial competition with sequential entry, Neven (1987) and Bonanno (1987) are the pioneering works, which have been extended to several directions (e.g., Loertscher and Muehlheusser, 2011).

${ }^{3} \mathrm{~A}$ good survey is presented by Chevalier-Roignant et al. (2011)

4 Nielsen (2002), Weeds (2002), Huisman and Kort (2003), and Pawlina and Kort (2006) study the IO problems within a real options framework, but with firms choosing only their timing in the models.
} 
substantially extend Ebina et al. (2015), which does not assume uncertainty on the growth path. Furthermore, we endogenize the leader's entry timing; this is also a significant extension of Ebina et al. (2015). We describe uncertainty as the product demand following a geometric Brownian motion, as in Pawlina and Kort (2006) and Huisman and Kort (2015). By doing so, we can analyze the effect of uncertainty on the two firms' entry timing and location strategies in a Hotelling model. Finally, for welfare analysis, we consider a situation in which a social planner locates two firms sequentially, whereas they compete and optimally set their prices to maximize their own profits after entry. Thus, we obtain implications from the viewpoint of social welfare by comparing the outcomes of the subgame perfect Nash equilibrium (hereafter SPNE) with those of social optimum for the planner.

The major findings of the study are as follows. First, the entry threshold of the follower firm is not monotonic in the volatility of the state variable. In the real options literature, the threshold is always an increasing function of volatility, irrespective of whether the firm is a leader or a follower if there is a negative externality 5 To the authors' best knowledge, this article is the first to show that greater uncertainty can lead to early entry of the follower firm in some cases. This finding indicates the importance of multiple dimensions for decision making. We also present some intuitive remarks on why non-monotonicity occurs.

Second, the leader firm optimally chooses the center as its location in case of low volatility, and the edge in case of high volatility. In addition, in case of high volatility, the delay of the follower's entry occurs in accordance with the maximum differentiation by the leader, contrasting with the standard intuition in which the leader's maximum differentiation induces the follower to enter earlier (e.g., Ebina et al., 2015). This is quite surprising in the context of spatial competition. Our paper is the first study to construct

\footnotetext{
${ }^{5}$ Mason and Weeds (2010) show that the threshold of a leader is non-monotonic if there is a positive externality. However, under the assumption of a negative externality, the thresholds of the leader and the follower are increasing in the volatility.
} 
a model in which demand evolves stochastically forever, and to examine the effect of uncertainty on locations within a continuous-time setting where both firms dynamically optimize their objectives $\left[{ }^{[}\right.$

Third, using welfare analysis, we numerically find that the social planner sets the leader's and follower's thresholds much lower and higher, respectively, than the sequential equilibrium in an autonomous economy. This finding indicates that the time interval between the two firms' optimal entry timings is narrower than that chosen by the social planner. Moreover, increasing uncertainty causes completely different effects on the locations and investment thresholds in an autonomous economy as well as in the social planner's choice. Thus, we conclude that uncertainty in the market may lead to a large social loss and that a policy might be needed from the viewpoint of welfare.

In summary, the analysis shows that multiple dimensions of strategies complicate the problem and may give different results from those in a single-dimension case, as shown in the real options literature.

The remaining part of the article is organized as follows. Section 2 sets up our model and formulates our problem. Section 3 derives the optimal timing and location of the follower firm. In Section 4, we first categorize the equilibrium type and then derive the optimal timing and location of the follower firm in a sequential equilibrium. We implement numerical calculations to examine how the leader's entry threshold and location are affected by exogenous parameters in Section 5. We also conduct welfare analysis and examine how social surplus is lost in an autonomous economy with two asymmetric firms in Section 6. Finally, some concluding remarks are presented in Section 7.

\footnotetext{
${ }^{6}$ In static frameworks, several studies investigate how uncertainty affects the optimal locations in the IO literature (e.g., Christou and Vettas, 2005, Meagher and Zauner, 2004).
} 


\section{The Model}

In this section, we construct our model based on Ebina et al. (2015) and introduce uncertainty into it as in Pawlina and Kort (2006).

Two firms, indexed by $i \in\{1,2\}$, produce homogeneous goods. Consumers are uniformly distributed over the unit segment $[0,1]$, as proposed by Hotelling (1929)! ? The density of consumer distribution at time $t$ is $Y_{t}$, which stochastically changes as explained later. Each consumer is indexed by $x \in[0,1]$ and repeatedly purchases at each instance $[t, t+d t)$ at most one unit of the good. He chooses the firm to purchase from when he actually decides to purchase. ${ }^{[}$The consumption of a unit of the good entails a positive utility. On the other hand, the consumer at point $x \in[0,1]$ incurs a quadratic transportation cost $c\left(x_{i}-x\right)^{2}$ and the price $p_{i t}$ at time $t \in[0, \infty)$ when buying a good from firm $i$ located at $x_{i} \in[0,1]$. To summarize, the utility of the consumer at point $x \in[0,1]$ at time $t \in[0, \infty)$ is given by

$$
u_{t}\left(x ; x_{1}, x_{2}, p_{1 t}, p_{2 t}\right)= \begin{cases}\bar{u}-p_{1 t}-c\left(x_{1}-x\right)^{2} & \text { if purchased from firm } 1 \\ \bar{u}-p_{2 t}-c\left(x_{2}-x\right)^{2} & \text { if purchased from firm } 2 \\ 0 & \text { otherwise }\end{cases}
$$

where $\bar{u}$ denotes the gross surplus each consumer enjoys from purchasing the good and $c$ is a parameter describing the level of transportation cost or product differentiation. The following assumption can make the equilibrium meaningful 9

Assumption 1. (i) $\bar{u}>3 c$. (ii) $c \geq 1$.

Now, we introduce uncertainty into our market model. The density of consumer distribution, or the market size $Y_{t}$, is dynamically stochastic. We impose the following assumption on $Y$.

\footnotetext{
7 This setting and the following assumptions are standard in the literature on spatial economics.

${ }^{8}$ In equilibrium, all consumers purchase a unit of the product at all times, according to Assumption 1 presented below.

9 Assumption 1 guarantees that at least one of the two firms has an incentive to supply a positive amount of goods after maximizing its profit wherever it is located.
} 
Assumption 2. Process $Y$ follows a geometric Brownian motion as

$$
\mathrm{d} Y_{t}=\alpha Y_{t} \mathrm{~d} t+\sigma Y_{t} \mathrm{~d} W_{t}
$$

where $\alpha$ is the expected growth rate, $\sigma$ is the market volatility, and $\left\{W_{t}\right\}_{t \geq 0}$ is a standard Brownian motion. The initial value of the state process, $Y_{0} \equiv y_{0}$, is sufficiently low.

Assumption 2 states that the future profit flow of each firm is uncertain and follows a geometric Brownian motion.10 The assumption on the initial value $y_{0}$ is standard in the real options literature, and means that the market is too small and that neither firm has made an entry into the market at the initial time.

The game in this article proceeds as follows. Firm $i$ chooses the time of entry $T_{i} \in$ $[0, \infty)$ and the location $x_{i} \in[0,1]$ simultaneously. The entry incurs an irreversible cost $F_{i}$ at $T_{i}$. Existing firm(s) simultaneously choose the price $p_{i t}$ at each time $t$, observing all available information such as the realization of $Y$ and their location(s) $x_{i}$. Although firm $i$ can vary the price $p_{i t}$ at any time, its location is fixed forever after the determination of $x_{i}$. We consider the Nash equilibrium in which firm $i$ maximizes its present value of cash flows with respect to $\left(T_{i}, x_{i},\left\{p_{i t}\right\}_{t \geq T_{i}}\right)$ given the other firm's strategy.

We present the following assumptions for the entry cost $F_{i}$.

Assumption 3. $F_{1}=F<\kappa F=F_{2}$, where $\kappa>1$.

Assumption 3 states that firm 1 has an advantage in the entry cost over firm 2 . Because asymmetry occurs only in the cost, we conjecture that firm 1 is always the leader for market entry in our model. Superscripts $\ell$ and $f$ represent the leader and the follower, respectively.

Now, let us describe the present value of the firms at time $t \in[0, \infty)$ given that firm $j$ enters the market at point $x_{j}$ at time $t=T_{j}$. Here, $T_{i}^{\ell}$ denotes the entry time of firm $i$

10 Exponential market growth is seen to be valid in many industries; for example, see Lages and Fernandes (2005) on telecommunication services, Victor and Ausubel (2002) on DRAM, and Vakratsas and Kolsarici (2008) on pharmaceuticals. 
when it is the leader, and $T_{j}^{f}$ denotes that of firm $j$ when it is the follower. As the model is time-homogeneous, the optimal entry time is expressed as the first hitting time; that is,

$$
T_{i}\left(y_{i}^{\ell}\right)=\inf \left\{t \geq 0 ; Y_{t} \geq y_{i}^{\ell}\right\} \quad \text { and } \quad T_{j}\left(y_{j}^{f}\right)=\inf \left\{t \geq 0 ; Y_{t} \geq y_{j}^{f}\right\}
$$

where $y_{i}^{\ell}$ and $y_{j}^{f}$ are the thresholds of the leader and the follower, respectively.

Let $\bar{x} \in[0,1]$ be a point at which the consumer is indifferent between purchasing from firm 1 and purchasing from 2. We can easily verify from (1) that

$$
\bar{x}=\frac{p_{2 t}-p_{1 t}+c\left(x_{2}^{2}-x_{1}^{2}\right)}{2 c\left(x_{2}-x_{1}\right)},
$$

which indicates that the optimal price of each firm depends on the locations of both firms $\left(x_{1}, x_{2}\right)$. Therefore, the value function of firm $j$ at time $t$ when it is the follower is written with discount rate $r$ as

$$
V_{j}^{f}\left(y ; y_{j}^{f}, x_{i}^{\ell}, x_{j}^{f}, p_{i t}^{\ell}, p_{j t}^{f}\right)=\mathbb{E}_{y}\left[\int_{T_{j}\left(y_{j}^{f}\right)}^{\infty} \mathrm{e}^{-r(s-t)} Y_{s} \int_{\bar{x}}^{1} p_{j s}^{f} \mathrm{~d} x \mathrm{~d} s-\mathrm{e}^{-r\left(T_{j}\left(y_{j}^{f}\right)-t\right)} F_{j}\right],
$$

where $\mathbb{E}_{y}$ denotes the expectation operator conditional on $Y_{t}=y$. We assume that $r>\alpha$ to ensure finiteness of the value function.11

On the other hand, the value function of firm $i$ as the leader, denoted by $V_{i}^{\ell}$, is expressed as

$$
\begin{aligned}
& V_{i}^{\ell}\left(y ; y_{i}^{\ell}, y_{j}^{f}, x_{i}^{\ell}, x_{j}^{f}, p_{i t}^{\ell}, p_{j t}^{f}\right) \\
& =\mathbb{E}_{y}\left[\int_{T_{i}\left(y_{i}^{\ell}\right)}^{T_{j}\left(y_{j}^{f}\right)} \mathrm{e}^{-r(s-t)} Y_{s} \int_{0}^{1} p_{i t}^{\ell} \mathrm{d} x \mathrm{~d} s+\int_{T_{j}\left(y_{j}^{f}\right)}^{\infty} \mathrm{e}^{-r(s-t)} Y_{s} \int_{0}^{\bar{x}} p_{i t}^{\ell} \mathrm{d} x \mathrm{~d} s-\mathrm{e}^{-r\left(T_{i}\left(y_{i}^{\ell}\right)-t\right)} F_{i}\right] .
\end{aligned}
$$

The first term in the right-hand side of (44) describes the discounted cash flow whereas firm $i$ is the monopolist, and the second term is the discounted cash flow after the other firm's entry. More concretely, if firm $i$ is the leader, it earns a monopoly profit flow for $t \in\left[T_{i}, T_{j}\right)$ and a duopoly profit flow for $t \geq T_{j}$.

\footnotetext{
${ }^{11}$ If $r \leq \alpha$, the integral of equation (3) diverges to positive infinity by choosing a larger time $T_{2}$, meaning that waiting for a longer time would always be a better strategy, and an optimal entry timing would not exist.
} 


\section{Follower's Value Functions}

In this section, we derive the optimal price, location, and timing outcomes of the follower firm. As in the literature, we implement backward induction for the derivation of the solutions. First, given the locations $x_{1}$ and $x_{2}$, we consider the problem of optimal prices at each time $t$ before and after the entry of firm 2 .

From (2), we can conclude that the uncertainty of $Y$ does not affect the equilibrium prices. Therefore, we obtain the following lemma, which describes the equilibrium prices of both firms. We omit the proof because the derivation is exactly the same as for Lemma 1 of Ebina et al. (2015).

Lemma 1. The prices set by the leader firm $i$ and the follower firm $j(\neq i)$ are, respectively,

$$
\begin{aligned}
& \hat{p}_{i t}^{\ell}= \begin{cases}p_{i}^{M}\left(x_{i}\right)=\bar{u}-c\left(1-x_{i}\right)^{2}, & t \in\left[T_{i}, T_{j}\right), \\
p_{i}^{D \ell}\left(x_{i}, x_{j}\right)=\frac{c}{3}\left(x_{j}-x_{i}\right)\left(2+x_{i}+x_{j}\right), & t \in\left[T_{j}, \infty\right),\end{cases} \\
& \hat{p}_{j t}^{f}=p_{j}^{D f}\left(x_{j}, x_{i}\right)=\frac{c}{3}\left(x_{j}-x_{i}\right)\left(4-x_{i}-x_{j}\right), \quad t \in\left[T_{j}, \infty\right) .
\end{aligned}
$$

Suppose that firm $i$ is the leader. Because the population of consumers on the unit interval is symmetric at $1 / 2$, we can assume $x_{i} \leq 1 / 2$ without loss of generality. Moreover, firm $i$ has monopolistic power over the price at $t \in\left[T_{i}, T_{j}\right)$ such that all consumers purchase its good. Then, the optimal price of firm $i$ before the entry of firm $j$ is the price at which the consumer at location 1 is indifferent between purchasing and not purchasing the good. The optimal monopolistic price $p_{i}^{M}$ in Lemma 1 satisfies this condition. The optimal price for the duopoly $p_{i}^{D}(i=1,2)$ is based on the standard calculation in the context of spatial competition (e.g., d'Aspremont et al., 1979)).

With the prices $\hat{p}_{i t}^{\ell}$ and $\hat{p}_{j t}^{f}$, the instantaneous profit flows of the two firms are expressed 
as $Y_{t} \pi_{i t}^{\ell}$ and $Y_{t} \pi_{j t}^{f}$, respectively, where

$$
\begin{aligned}
& \pi_{i t}^{\ell}\left(x_{i}, x_{j}\right)= \begin{cases}\pi_{i}^{M \ell}\left(x_{i}\right)=\bar{u}-c\left(1-x_{i}\right)^{2}, & t \in\left[T_{i}, T_{j}\right), \\
\pi_{i}^{D \ell}\left(x_{i}, x_{j}\right)=\frac{c}{18}\left(x_{j}-x_{i}\right)\left(2+x_{i}+x_{j}\right)^{2}, & t \in\left[T_{j}, \infty\right),\end{cases} \\
& \pi_{j t}^{f}\left(x_{i}, x_{j}\right)= \begin{cases}0, & t \in\left[0, T_{j}\right), \\
\pi_{j}^{D f}\left(x_{i}, x_{j}\right)=\frac{c}{18}\left(x_{j}-x_{i}\right)\left(4-x_{i}-x_{j}\right)^{2}, & t \in\left[T_{j}, \infty\right)\end{cases}
\end{aligned}
$$

as $\bar{x}=\left(2+x_{1}+x_{2}\right) / 6$ in equilibrium.

Suppose that the current time is 0 and firm $i$ enters the market. The net present values of firm $i$ when it is the leader and when it is the follower, including the option value of the future profit flows, are expressed as, respectively,

$$
V_{i}^{\ell}\left(y ; y_{j}^{f}, x_{i}, x_{j}\right)=\mathbb{E}_{y}\left[\int_{t}^{T_{j}\left(y_{j}^{f}\right)} \mathrm{e}^{-r(s-t)} \pi_{i}^{M \ell}\left(x_{i}\right) Y_{s} \mathrm{~d} s+\int_{T_{j}\left(y_{j}^{f}\right)}^{\infty} \mathrm{e}^{-r(s-t)} \pi_{i}^{D \ell}\left(x_{i}, x_{j}\right) Y_{s} \mathrm{~d} s-F_{i}\right]
$$

and

$$
V_{j}^{f}\left(y ; y_{j}^{f}, x_{i}, x_{j}\right)=\mathbb{E}_{y}\left[\int_{T_{j}\left(y_{j}^{f}\right)}^{\infty} \mathrm{e}^{-r(s-t)} \pi_{j}^{D f}\left(x_{i}, x_{j}\right) Y_{s} \mathrm{~d} s-\mathrm{e}^{-r\left(T_{j}\left(y_{j}^{f}\right)-t\right)} F_{j}\right]
$$

\subsection{Optimal location and entry threshold}

First, we consider the problem of the follower relating to when it enters and where it locates in the market. We assume that firm $i$ is the leader and has already invested and located at $x_{i} \in[0,1 / 2]$. We write the value function of the follower as

$$
\hat{V}_{j}^{f}\left(y ; y_{i}^{\ell}, x_{i}\right)=\max _{y_{j}^{f}, x_{j}} V_{j}^{f}\left(y ; y_{j}^{f}, x_{i}, x_{j}\right)
$$

Note that the function $\hat{V}_{j}^{f}$ depends on the other firm's location as the instantaneous profit flow $\pi_{j}^{D f}$ depends on $x_{i}$. This implies that the pair of optimal strategies $\left(y_{j}^{f}, x_{j}\right)$ also depends on $x_{i}$. However, with regard to the location of the follower firm $j$, we have the following lemma.

Lemma 2. In equilibrium, the follower firm $j$ always locates at $x_{j}^{f}=1$. 
Proof. Suppose that $Y_{t}=y_{j}^{f}$. From (8) and (10), by differentiating $V_{j}^{f}$ with respect to $x_{j}$, we have

$$
\left.\frac{\partial V_{j}^{f}}{\partial x_{j}}\left(y ; y_{j}^{f}, x_{i}, x_{j}\right)\right|_{y=y_{f}^{j}}=\frac{1}{r-\alpha} \frac{c}{18}\left(4+x_{1}-3 x_{2}\right)\left(4-x_{1}-x_{2}\right)
$$

which is positive for any $\left(x_{i}, x_{j}\right) \in[0,1]^{2}$ when $r-\alpha>0$ and $c>0$. Thus, the optimal location of firm $j$ should be $x_{j}^{f}=1$ when it is the follower.

Once the optimal location is obtained, the optimal timing can easily be derived as in the following lemma. We omit the proof because it is an easy exercise.

Lemma 3. The value function of firm $j$ as the follower is given by

$$
\hat{V}_{j}^{f}\left(y ; x_{i}\right)= \begin{cases}\left(\frac{y_{j}^{f}\left(x_{i}\right) \pi_{j}^{D f}\left(x_{i}, 1\right)}{r-\alpha}-F_{j}\right)\left(\frac{y}{y_{j}^{f}\left(x_{i}\right)}\right)^{\beta}, & \text { if } y<y_{j}^{f}\left(x_{i}\right), \\ \frac{y \pi_{j}^{D f}\left(x_{i}, 1\right)}{r-\alpha}-F_{j}, & \text { if } y \geq y_{j}^{f}\left(x_{i}\right),\end{cases}
$$

where

$$
\beta=\frac{1}{2}-\frac{\alpha}{\sigma^{2}}+\sqrt{\left(\frac{1}{2}-\frac{\alpha}{\sigma^{2}}\right)^{2}+\frac{2 r}{\sigma^{2}}}
$$

and the investment threshold of the follower is

$$
y_{j}^{f}\left(x_{i}\right)=\frac{\beta}{\beta-1} \frac{(r-\alpha)}{\pi_{j}^{D f}\left(x_{i}, 1\right)} F_{j} .
$$

From Lemma 2, the follower firm always locates as far away from the location of the leader as possible when entering the market. This replicates the results of Lambertini (2002) and Ebina et al. (2015) and seems to be robust to the endogeneity of the follower's entry timing under uncertainty.

From (11), we easily obtain the following corollary:

Corollary 1. If $x_{1}$ is increased, the optimal threshold for the follower to enter $y_{2}^{f}\left(x_{1}\right)$ is increased. 
The corollary shows that the leader can obstruct the follower's entry by choosing its location close to $1 / 2$. On the other hand, setting $x_{i}$ close to $1 / 2$ can induce tougher price competition after the follower's entry. The leader firm faces the trade-off between the two with regard to location.

\section{Leader's Strategy and Equilibria}

In this section, we derive the outcome of subgame Nash equilibrium. We face two difficulties when deriving the equilibria, compared to the process used in Pawlina and Kort (2006). The first difficulty arises because the value functions of our model are determined by the firms' entry timings as well as location choices, but in the previous study, these functions are determined by only their entry timings. Thus, the location choice in our model entails complexity, making it difficult to derive an equilibrium.

The second difficulty arises from the asymmetry of the firms' profits. Because the firms' location choices may not be symmetric on the Hotelling interval $[0,1]$, the profits $\pi_{i}^{D l}\left(x_{i}, 1\right)$ and $\pi_{j}^{D f}\left(x_{i}, 1\right)$ may have different values, leading to a situation where an equilibrium becomes more complicated to derive.

To derive an equilibrium, we need to consider the leader's optimization problem given the follower's strategy. Suppose that firm $i$ immediately enters the market with location $x_{i}$. Given the other firm's strategy $\left(y_{j}, x_{j}\right)=\left(y_{j}^{f}\left(x_{i}\right), 1\right)$, the present value of the leader firm $i(9)$ is

$$
V_{i}^{\ell}\left(y ; y_{j}^{f}\left(x_{i}\right), x_{i}, 1\right)= \begin{cases}\frac{y \pi_{i}^{M}\left(x_{i}\right)}{r-\alpha}-F_{i}-\frac{y_{j}^{f}\left(\pi_{i}^{M}\left(x_{i}\right)-\pi_{i}^{D \ell}\left(x_{i}, 1\right)\right)}{r-\alpha}\left(\frac{y}{y_{j}^{f}\left(x_{i}\right)}\right)^{\beta} & \text { for } y \leq y_{j}^{f}\left(x_{i}\right), \\ \frac{y \pi_{i}^{D \ell}\left(x_{i}, 1\right)}{r-\alpha}-F_{i} & \text { for } y>y_{j}^{f}\left(x_{i}\right) .\end{cases}
$$

The firm chooses its location $x_{i}$ such that (12) is maximized. Thus, we write

$$
\tilde{V}_{i}^{\ell}(y)=\max _{x_{i} \in[0,1 / 2]} V_{i}^{\ell}\left(y ; y_{j}^{f}\left(x_{i}\right), x_{i}, 1\right) \quad \text { and } \quad \tilde{x}_{i}^{\ell}(y)=\underset{x_{i} \in[0,1 / 2]}{\operatorname{argmax}} V_{i}^{\ell}\left(y ; y_{j}^{f}\left(x_{i}\right), x_{i}, 1\right) .
$$

Now, we investigate the outcome of the SPNE. Following Pawlina and Kort (2006), three types of equilibria occur: preemptive, sequential, and simultaneous. The leader's 
location choice and threshold are key to investigate the equilibrium, because the follower's strategy for location and entry timing is dominant in that the follower chooses its location $x_{j}=1$ and threshold $y_{j}^{f}$ after the leader enters the market.

\subsection{Simultaneous equilibrium}

First, we consider a simultaneous equilibrium and show that it cannot occur.

Lemma 4. A simultaneous equilibrium cannot occur as an outcome of SPNE.

Proof. Proposition 3 in Pawlina and Kort (2006) presents the condition for a simultaneous equilibrium to occur. According to the proposition, the parameter $\kappa$ that determines the type of equilibrium should be smaller than some threshold $\kappa^{* *}$. In our setting, we can easily show that $\kappa^{* *}=1$ and $\kappa>1$, thus completing the proof.

Intuitively, when there is no cash flow in advance of the entry, the two firms have no incentive to coordinate under cost asymmetry, and hence a simultaneous equilibrium cannot occur.

\subsection{Preemptive equilibrium}

Second, we consider the second type of equilibrium, preemption equilibrium. Pawlina and Kort (2006) define a preemptive equilibrium as the situation in which firm 2 , which is disadvantaged in an investment cost, has an incentive to become the leader. In this case, firm 1 needs to note that firm 2 would enter the market before the state variable $Y$ for firm 1 reaches the optimal threshold.

Let $y_{21}^{P}\left(x_{1}\right)$ be the lowest level of the state variable $y$ for which firm 2 is indifferent between being the leader and being the follower, given firm 1's location $x_{1}$. Formally, $y_{i j}^{P}\left(x_{j}\right)$ is the smallest solution to $\xi_{i}\left(y ; x_{j}\right)=0$, where $\xi_{i}\left(y ; x_{j}\right)$ is defined as

$$
\xi_{i}\left(y ; x_{j}\right) \equiv \tilde{V}_{i}^{\ell}(y)-\hat{V}_{i}^{f}\left(y ; x_{j}\right)
$$


and $\tilde{V}_{i}^{\ell}(y)$ and $\hat{V}_{i}^{f}$ are given by equations (13) and (10), respectively. Intuitively, a positive $\xi_{i}$ indicates that firm $i$ prefers immediate entry as the leader to waiting to be the follower. Note that $\xi_{i}$ is already maximized with respect to its location $x_{i}$, but this depends on the other firm's location $x_{j}$.

Now, suppose that $y_{21}^{P}\left(\tilde{x}_{1}^{\ell}\left(y_{1}^{S \ell}\right)\right)<y_{1}^{S \ell}$, where

$$
y_{1}^{S \ell}=\underset{y_{1}}{\operatorname{argmax}} \mathbb{E}_{y}\left[\mathrm{e}^{-r\left(T_{1}\left(y_{1}\right)-t\right)} \tilde{V}_{1}^{\ell}\left(y_{1}\right)\right] .
$$

For $y_{21}^{P}\left(\tilde{x}_{1}^{\ell}\left(y_{1}^{S \ell}\right)\right) \leq y<y_{1}^{S \ell}$, firm 1 should take one of the following two actions to deter the other firm's entry: (i) immediate investment, or (ii) change of location $x_{1}$. If firm 1 chooses an immediate investment, the location should be maximized at $y_{21}^{P}\left(\tilde{x}_{1}^{\ell}\left(y_{1}^{S \ell}\right)\right)$, such that $x_{1}$ can be expressed as

$$
\tilde{x}_{1}^{\ell}\left(y_{21}^{P}\left(\tilde{x}_{1}^{\ell}\left(y_{1}^{S \ell}\right)\right)\right)
$$

On the other hand, firm 1 can change the location $x_{1}$ to hinder the entry of firm 2 . As Corollary 1 shows, location $x_{1}$ should be increased. Therefore, firm 1 actually changes the location until the following inequality holds for $x_{1} \neq 0$ :

$$
\frac{\partial}{\partial x_{1}} \mathbb{E}_{y}\left[\mathrm{e}^{-r\left(T_{1}\left(y_{21}^{P}\left(x_{1}\right)\right)-t\right)} \tilde{V}_{1}^{\ell}\left(y_{21}^{P}\left(x_{1}\right)\right)\right]<0 .
$$

From the above observation, a preemptive equilibrium is rather complicated and seems hard to solve, even numerically. For example, $\tilde{x}_{1}^{\ell}$ in (15) is solved through the maximization (13) and non-linear equation (14). One reason for the difficulty is that the payoff of a Hotelling-type model is a cubic function with respect to the state variable ${ }^{12}$ Hence, we assume that only a sequential equilibrium can occur, and that a cost-advantaged firm can optimally choose its entry timing and location given the other firm's strategy. It is worth arguing that our model is still applicable to many realistic situations, for example, to a market where one firm has a large cost advantage over the other. Therefore, we impose the following assumption to eliminate the possibility of a preemptive equilibrium.

\footnotetext{
${ }^{12}$ On the contrary, Huisman and Kort (2015) assume a quadratic payoff function, which allows them to explicitly solve a preemptive equilibrium.
} 
Assumption 4. The order of entries is exogenously determined. More concretely, firm 1 is always the leader, with firm 2 as the follower.

If the cost asymmetry is substantial, there is only a sequential equilibrium. Pawlina and Kort (2006) show the necessary and sufficiently condition for Assumption 4.

\subsection{Sequential equilibrium}

Now, we consider the third type of equilibrium, sequential equilibrium. In the following, we derive the optimal threshold for firm 1 as the leader under Assumption 4.

Let $T_{i}$ denote the timing at which the leader, firm 1 , enters when the state variable $Y$ first touches $\bar{y}_{1}^{\ell}$,

$$
T_{1}\left(\bar{y}_{1}^{\ell}\right)=\inf \left\{t \geq 0 ; Y_{t}=\bar{y}_{1}^{\ell}\right\}
$$

Then, the option value of the entry for $y<\bar{y}_{1}^{\ell}$ with location $x_{1}$ is given by

$$
\begin{aligned}
\hat{V}_{1}^{\ell}\left(y ; x_{1}\right) & =\mathbb{E}_{y}\left[\mathrm{e}^{-r\left(T_{1}\left(\bar{y}_{1}^{\ell}\right)-t\right)} \tilde{V}_{1}^{\ell}\left(\bar{y}_{1}^{\ell} ; x_{1}\right)\right] \\
& =\left(\frac{\pi_{1}^{M \ell}\left(x_{1}\right) \bar{y}_{1}^{\ell}}{r-\alpha}-\frac{\left[\pi_{1}^{M \ell}\left(x_{1}\right)-\pi_{1}^{D \ell}\left(x_{1}, 1\right)\right] y_{2}^{f}\left(x_{1}\right)}{r-\alpha}\left(\frac{\bar{y}_{1}^{\ell}}{y_{2}^{f}\left(x_{1}\right)}\right)^{\beta}-F_{i}\right)\left(\frac{y}{\bar{y}_{1}^{\ell}}\right)^{\beta} .
\end{aligned}
$$

Thus, the threshold of firm 1 that maximizes the above expression with respect to $\bar{y}_{1}^{\ell}$ is

$$
\bar{y}_{1}^{S \ell}\left(x_{1}\right)=\frac{\beta}{\beta-1} \frac{r-\alpha}{\pi_{1}^{M \ell}\left(x_{1}\right)} F_{1} .
$$

Note that $\bar{y}_{1}^{S \ell}\left(x_{1}\right)$ does not explicitly depend on the other firm's strategy $\left(y_{2}, x_{2}\right)$. By substituting $y_{1}^{S \ell}\left(x_{1}\right)$ into the first line of (12), we have the value function of the leader at the sequential equilibrium before deciding its location as follows:

$$
\begin{aligned}
\widehat{V}_{1}^{\ell}\left(x_{1}\right) & =\hat{V}_{1}^{\ell}\left(\bar{y}_{1}^{S \ell}\left(x_{1}\right) ; x_{1}\right)=\frac{\pi_{1}^{M \ell}\left(x_{1}\right) \bar{y}_{1}^{S \ell}}{r-\alpha}-\frac{\left[\pi_{1}^{M \ell}\left(x_{1}\right)-\pi_{1}^{D \ell}\left(x_{1}\right)\right] y_{2}^{f}\left(x_{1}\right)}{r-\alpha}\left(\frac{\bar{y}_{1}^{S \ell}}{y_{2}^{f}\left(x_{1}\right)}\right)^{\beta}-F_{1} \\
& =\frac{F_{1}}{\beta-1}-\frac{\beta}{\beta-1} \frac{\pi_{1}^{M \ell}\left(x_{1}\right)-\pi_{1}^{D \ell}\left(x_{1}\right)}{\pi_{2}^{D f}\left(x_{1}\right)}\left(\frac{\pi_{2}^{D f}\left(x_{1}\right)}{\pi_{1}^{M \ell}\left(x_{1}\right)} \frac{F_{1}}{F_{2}}\right)^{\beta} F_{2} .
\end{aligned}
$$


The optimal location for the leader firm is expressed as

$$
x_{1}^{S \ell}=\underset{x_{1} \in[0,1 / 2]}{\operatorname{argmax}} \widehat{V}_{1}^{\ell}\left(x_{1}\right)=\underset{x_{1} \in[0,1 / 2]}{\operatorname{argmin}} \psi_{1}\left(x_{1} ; \beta\right),
$$

where

$$
\psi_{1}\left(x_{1} \beta\right):=\frac{\pi_{1}^{M \ell}\left(x_{1}\right)-\pi_{1}^{D \ell}\left(x_{1}\right)}{\pi_{2}^{D f}\left(x_{1}\right)}\left(\frac{\pi_{2}^{D f}\left(x_{1}\right)}{\pi_{1}^{M \ell}\left(x_{1}\right)}\right)^{\beta} .
$$

Now, we have the following lemma.

Lemma 5. There exists a pair $(\underline{\beta}, \bar{\beta})$ such that

(i) $x_{1}^{S \ell}=1 / 2$ for $\beta>\bar{\beta}$,

(ii) $x_{1}^{S \ell}=0$ for $\beta<\underline{\beta}$.

Proof. Consider the function $\log \psi_{1}$. By differentiating it, we have

$$
\frac{\mathrm{d}}{\mathrm{d} x_{1}} \log \psi_{1}\left(x_{1} ; \beta\right)=\frac{\pi_{1}^{M \ell \ell}\left(x_{1}\right)-\pi_{1}^{D \ell \prime}\left(x_{1}\right)}{\pi_{1}^{M \ell}\left(x_{1}\right)-\pi_{1}^{D \ell}\left(x_{1}\right)}-\frac{\pi_{2}^{D f^{\prime}}\left(x_{1}\right)}{\pi_{2}^{D f}\left(x_{1}\right)}+\beta\left(\frac{\pi_{2}^{D f^{\prime}}\left(x_{1}\right)}{\pi_{2}^{D f}\left(x_{1}\right)}-\frac{\pi_{1}^{M \ell \prime}\left(x_{1}\right)}{\pi_{1}^{M \ell}\left(x_{1}\right)}\right) .
$$

Because $\pi_{2}^{D f^{\prime}}\left(x_{1}\right) / \pi_{2}^{D f}\left(x_{1}\right)-\pi_{1}^{M \ell \prime}\left(x_{1}\right) / \pi_{1}^{M \ell}\left(x_{1}\right)$ is strictly negative, (20) is also negative for any $x_{1} \in[0,1 / 2]$ if $\beta$ is sufficiently large. We verify the first part from the fact that $\log$ is a monotonic function. We obtain the second part by noting that

$$
\begin{aligned}
\lim _{\beta \searrow 1} \frac{\mathrm{d}}{\mathrm{d} x_{1}} \log \psi_{1}\left(x_{1} ; \beta\right) & =\frac{\pi_{1}^{M \ell \ell}\left(x_{1}\right)-\pi_{1}^{D \ell \prime}\left(x_{1}\right)}{\pi_{1}^{M \ell}\left(x_{1}\right)-\pi_{1}^{D \ell}\left(x_{1}\right)}-\frac{\pi_{1}^{M \ell \prime}\left(x_{1}\right)}{\pi_{1}^{M \ell}\left(x_{1}\right)} \\
& =\frac{\pi_{1}^{M \ell \prime}\left(x_{1}\right) \pi_{1}^{D \ell}\left(x_{1}\right)-\pi_{1}^{M \ell}\left(x_{1}\right) \pi_{1}^{D \ell \prime}\left(x_{1}\right)}{\pi_{1}^{M \ell}\left(x_{1}\right)\left(\pi_{1}^{M \ell}\left(x_{1}\right)-\pi_{1}^{D \ell}\left(x_{1}\right)\right)}
\end{aligned}
$$

which is positive for any $x_{1} \in[0,1 / 2]$.

Note that parameter $\beta$ is monotonically decreasing in the volatility parameter $\sigma$; that is, $\partial \beta / \partial \sigma<0$. The above proposition implies that in a sequential equilibrium, the location of the leader is the center if the volatility of the market size is sufficiently small. On the other hand, if the uncertainty over the future size of the market is sufficiently 
large, the optimal location of the leader in a sequential equilibrium moves closer to the edge. We can derive similar results for $\alpha$ and $r$, the expected growth rate of the market size and the discount rate, because $\partial \beta / \partial \alpha<0$ and $\partial \beta / \partial r>0$.

Proposition 1. (i) There exists a pair $(\underline{\sigma}, \bar{\sigma})$ such that

(a) $x_{1}^{S \ell}=1 / 2$ for $\sigma<\underline{\sigma}$,

(b) $x_{1}^{S \ell}=0$ for $\sigma>\bar{\sigma}$.

(ii) There exists a pair $(\underline{\alpha}, \bar{\alpha})$ such that

(a) $x_{1}^{S \ell}=1 / 2$ for $\alpha<\underline{\alpha}$,

(b) $x_{1}^{S \ell}=0$ for $\alpha>\bar{\alpha}$.

(iii) There exists a pair $(\underline{r}, \bar{r})$ such that

(a) $x_{1}^{S \ell}=0$ for $r<\underline{r}$,

(b) $x_{1}^{S \ell}=1 / 2$ for $r>\bar{r}$.

In what follows, we present some intuitive explanation on the relationship between $\sigma$ and $x_{1}^{S \ell}$. We have already obtained the value function of the leader as (17). Through calculations, we interpret the first term of (17) as the present value of the monopolistic profit flows and the second term as the negative option value associated with the other firm's entry. Note that the first term is totally independent of the leader's location. Thus, the leader firm chooses the optimal location $x_{1}$ such that the negative option value is minimized. In other words, $x_{1}$ is a minimizer of $\psi_{1}$ defined by (19).

We find that $\psi_{1}$ consists of two terms, $\left(\pi_{1}^{M \ell}\left(x_{1}\right)-\pi_{1}^{D \ell}\left(x_{1}\right)\right) / \pi_{2}^{D f}\left(x_{1}\right)$ and $\left(\pi_{2}^{D f}\left(x_{1}\right) F_{1} / \pi_{1}^{M \ell}\left(x_{1}\right) F_{2}\right)^{\beta}$. The first term represents the change in profit flows induced by the follower's entry, or the negative effect on the leader's profit flows when the follower actually enters the market. Of course, this term is increasing in $x_{1}$, indicating that $x_{1}$ should be lower if firm 1 takes this effect into account. On the other hand, the second term represents the expected time 
of the follower firm's entry. More concretely, the term is proportional to $\mathbb{E}\left[\mathrm{e}^{-r T_{2}}\right]$. This term is decreasing in $x_{1}$ because $\pi_{1}^{M \ell \prime}>0$ and $\pi_{2}^{D f \prime}<0$. In other words, firm 1 can deter the entry of firm 2 by a high value of $x_{1}$. As a whole, to minimize $\psi_{1}$, firm 1 needs to consider the trade-off.

Suppose that $\sigma$ is sufficiently large. Then, $\beta$ is close to 1 and the effect of $x_{1}$ on the follower's entry time is quite marginal. In this case, the first effect dominates the second one, indicating that firm 1 optimally chooses $x_{1}^{S \ell}=0$ as its location. The story is the opposite for a small $\sigma$. In this case, $\beta$ is significantly large and thus firm 1 can hinder the entry of firm 2 by choosing its location as close to the center as possible. In other words, the second effect dominates the first one and the optimal location is given by $1 / 2$.

In the standard real options literature, most studies consider the case of only the optimal entry timing. Then, $\sigma$ affects $\psi_{1}$ only through $\beta$ and always has a positive effect on the value function of the leader (17). However, under our setting, where each firm chooses the timing as well as location, the problem is not simple, with results differing from those in the standard model. In fact, Section 5 presents a novel result due to the multi-dimensionality of strategies.

Finally, by substituting $x_{1}^{S \ell}$ for each case in Proposition 1, we have the following proposition stating the outcomes of SPNE.

Proposition 2. Let $\left(y_{1}^{E \ell}, y_{2}^{E f}, x_{1}^{E \ell}, x_{2}^{E f}, p_{1}^{E \ell}, p_{2}^{E f}\right)$ be the equilibrium solution of the locations, thresholds, and prices. Then, we have the following three cases.

(a) If Equation (20) is negative for any $x_{1} \in[0,1 / 2]$, the outcome of the subgame perfect equilibrium is expressed as $\left(y_{1}^{E \ell}, y_{2}^{E f}, x_{1}^{E \ell}, x_{2}^{E f}, p_{1}^{E \ell}, p_{2}^{E f}\right)=\left(y_{1}^{*}, y_{2}^{*}, x_{1}^{*}, x_{2}^{*}, p_{1}^{*}, p_{2}^{*}\right)$, 
where

$$
\begin{aligned}
& y_{1}^{*} \equiv \frac{\beta}{\beta-1} \frac{4(r-\alpha) F}{4 \bar{u}-c}, y_{2}^{*} \equiv \frac{\beta}{\beta-1} \frac{144(r-\alpha) \kappa F}{25 c}, \\
& x_{1}^{*} \equiv \frac{1}{2}, x_{2}^{*} \equiv 1, p_{1 t}^{*} \equiv \begin{cases}p_{1}^{M}\left(x_{1}^{*}\right)=\bar{u}-\frac{c}{4} & \text { for } t \in\left[T_{1}\left(y_{1}^{*}\right), T_{2}\left(y_{2}^{*}\right)\right) \\
p_{1}^{D}\left(x_{1}^{*}, x_{2}^{*}\right)=\frac{7 c}{12} & \text { for } t \in\left[T_{2}\left(y_{2}^{*}\right), \infty\right),\end{cases} \\
& p_{2 t}^{*} \equiv p_{2}^{D}\left(x_{1}^{*}, x_{2}^{*}\right)=\frac{5 c}{12} \text { for } t \in\left[T_{2}\left(y_{2}^{*}\right), \infty\right) .
\end{aligned}
$$

The consumer at $\bar{x}^{*} \equiv \frac{7}{12}$ is indifferent between purchasing the good from firm 1 and purchasing it from firm 2.

(b) If Equation (20) is positive for any $x_{1} \in[0,1 / 2]$, the outcome of the subgame perfect equilibrium is expressed as $\left(y_{1}^{E \ell}, y_{2}^{E f}, x_{1}^{E \ell}, x_{2}^{E f}, p_{1}^{E \ell}, p_{2}^{E f}\right)=\left(y_{1}^{* *}, y_{2}^{* *}, x_{1}^{* *}, x_{2}^{* *}, p_{1}^{* *}, p_{2}^{* *}\right)$, where

$$
\begin{aligned}
& y_{1}^{* *} \equiv \frac{\beta}{\beta-1} \frac{(r-\alpha) F}{\bar{u}-c}, y_{2}^{* *} \equiv \frac{\beta}{\beta-1} \frac{2(r-\alpha) \kappa F}{c}, \\
& x_{1}^{* *} \equiv 0, x_{2}^{* *} \equiv 1, \\
& p_{1 t}^{* *} \equiv \begin{cases}p_{1}^{M}\left(x_{1}^{* *}\right)=\bar{u}-c & \text { for } t \in\left[T_{1}\left(x_{1}^{* *}\right), T_{2}\left(x_{2}^{* *}\right)\right) \\
p_{1}^{D}\left(x_{1}^{* *}, x_{2}^{* *}\right)=c & \text { for } t \in\left[T_{2}\left(x_{2}^{* *}\right), \infty\right),\end{cases} \\
& p_{2 t}^{* *} \equiv p_{2}^{D}\left(x_{1}^{* *}, x_{2}^{* *}\right)=c \text { for } t \in\left[T_{2}\left(x_{2}^{* *}\right), \infty\right) .
\end{aligned}
$$

The consumer at $\bar{x}^{* *}=\frac{1}{2}$ is indifferent between purchasing the good from firm 1 and purchasing it from firm 2.

Remark 1. We cannot analytically prove the presence of an inner solution

$$
\left(y_{1}^{E \ell}, y_{2}^{E f}, x_{1}^{E \ell}, x_{2}^{E f}, p_{1}^{E \ell}, p_{2}^{E f}\right)=\left(y_{1}^{* * *}, y_{2}^{* * *}, x_{1}^{* * *}, x_{2}^{* * *}, p_{1}^{* * *}, p_{2}^{* * *}\right)
$$

with $x_{1}^{* * *} \in(0,1 / 2)$ when $\underline{\beta}<\beta<\bar{\beta}$. Fortunately, from our numerical analysis, there exists an inner solution under a wide range of parameter settings if $\beta$ is intermediate and $\bar{u}$ is large enough. 
Proposition 2 shows three cases for the leader's location in our sequential equilibrium. Which case occurs depends on the parameters describing the dynamics of the market size $\alpha, \sigma$, and so on. To grasp the intuition behind the two propositions, we proceed with a numerical analysis in the next section.

\section{$5 \quad$ Numerical Analysis}

In this section, we investigate in depth the underlying properties of our model using numerical analysis. First, we investigate the parameter effects, especially $\sigma$, on firm 1's equilibrium location. Second, we derive the outcome of SPNE and discuss how the parameters influence the firms' behavior in equilibrium.

\subsection{Leader's equilibrium location}

This subsection numerically examines the effects of $\sigma$ and $\alpha$ on the leader's location $x_{1}^{E \ell}$. We set the parameters as $\bar{u}=30, c=1, r=0.1, \kappa=2$, and $F=10$. Figure 1 illustrates how the parameters $\sigma$ and $\alpha$ affect the equilibrium location of the leader.

First, we discuss the relationship between $x_{1}^{E \ell}$ and $\alpha$. From the figure, $x_{1}^{E \ell}=1 / 2$ always holds when $\alpha \leq 0.096$; this is depicted at the upper left-hand side. On the other hand, $x_{1}^{E}=0$ always holds when $\alpha \geq 0.099$, as depicted at the lower right-hand side for any $\sigma \in(0,1]$. Consequently, one finds that if the expected growth rate of the market size $\alpha$ is sufficiently large (small), the equilibrium location of firm $1, x_{1}^{E \ell}$, is always at the center (edge) for any $\sigma \in(0,1]$. If $\alpha$ takes an intermediate value, $x_{1}^{E \ell}$ is interior in the interval $(0,1 / 2)$. These results are consistent with the analytical properties shown in Proposition 1. Ebina et al. (2015) obtain a similar result with their numerical analysis in

pages 908-910. In addition, the figure illustrates that $x_{1}^{E \ell}$ is decreasing in $\alpha$ on the range $[0.0965,0.099]$. This observation confirms the existence of the inner solution mentioned in Remark 1 ,

Next, we discuss the relationship between $x_{1}^{E \ell}$ and $\sigma$. From the figure, $x_{1}^{E \ell}$ is decreasing 


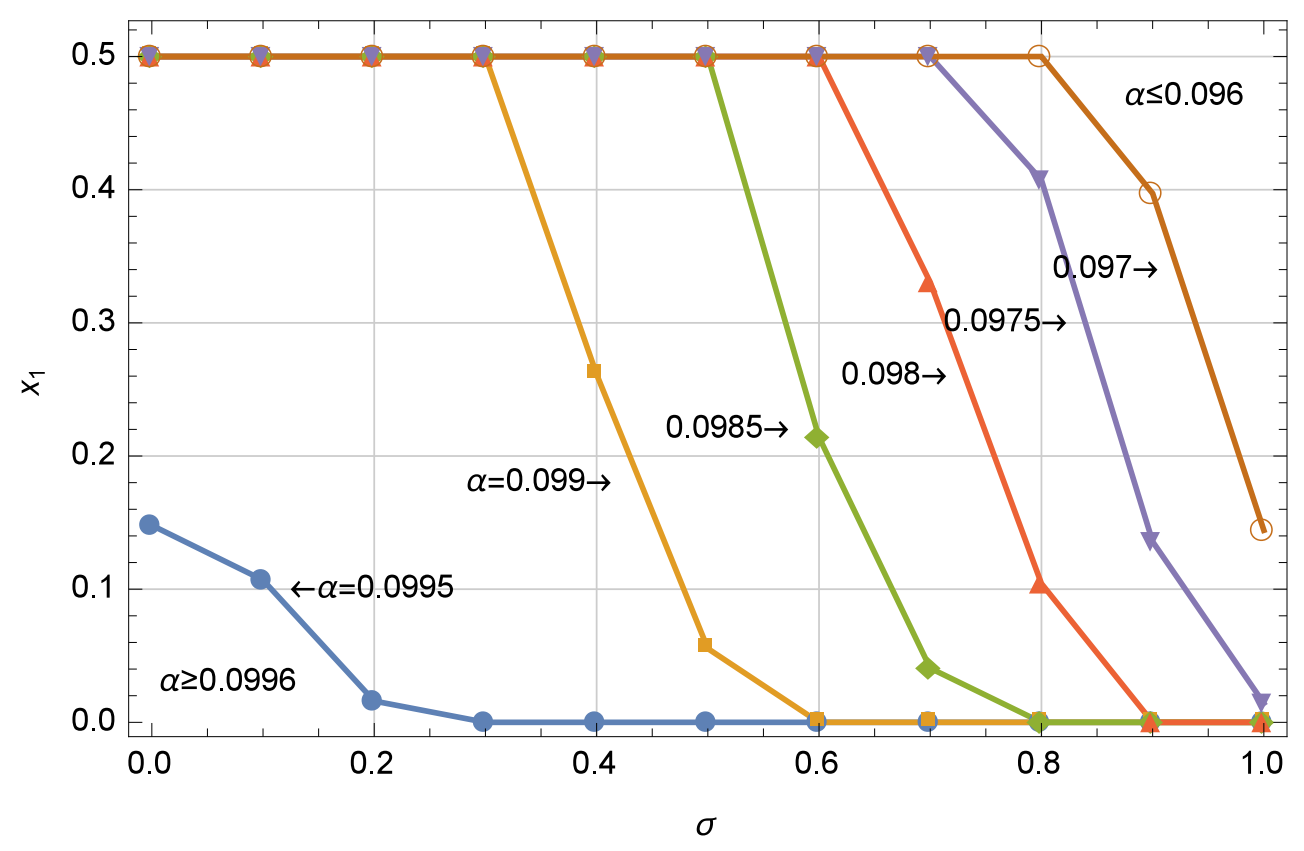

Figure 1: The relationship between $x_{1}^{E \ell}$ and $\alpha$, or $\sigma$.

in $\sigma$ and is between 0 and $1 / 2$ when $\sigma$ takes an intermediate value, again confirming the existence of an inner solution. Further, if $\alpha$ is sufficiently large (small), $x_{1}^{E \ell}$ is constant and equal to $0\left(1 / 2\right.$, respectively) for any $\sigma \in(0,1]$. These two facts imply that $x_{1}^{E \ell}$ is non-increasing in $\sigma$ for a wide variety of parameter settings.

We intuitively explain the above observation as follows. A large value of $\alpha$ means the rapid market size growth on average, inducing the follower to enter the market earlier. Then, the leader should avoid price competition with the follower. Thus, the leader firm optimally chooses $x_{1}^{E \ell}=0$.

On the other hand, Figure 1 confirms the analytical result presented in Proposition 2. If $\sigma$ is large, the entry deterrence through a higher $x_{1}$ is less effective, as explained in the previous section. In other words, the follower firm should have an incentive to wait longer irrespective of $x_{1}$ because the uncertainty in market size is significant. Thus, the leader firm optimally sets $x_{1}^{E \ell}$ lower for a small $\sigma$. 
In sum, we conclude that the leader's location in equilibrium is decreasing (or at least non-increasing) in $\sigma$ in most cases. Similar observations can be found for the effect of $\alpha$ and $r$.

\subsection{Subgame perfect Nash equilibrium}

Here, we investigate how the most significant parameter of this study, $\sigma$, affects the outcome of SPNE. We first set the parameters as $\bar{u}=30, c=1, r=0.1, \alpha=0.99, \kappa=2$, and $F=10$. Under this setting, the equilibrium is an inner solution for $\sigma \in[0.4,0.5]$. The outcomes of SPNE and the values of the leader and the follower at SPNE, $V_{1}^{E \ell} \equiv \hat{V}_{1}\left(y ; x_{1}^{E \ell}\right)$ and $\hat{V}_{2}^{E f}\left(y ; x_{1}^{E \ell}\right)$, are presented in Table 1 for $\sigma \in\{0.0001,0.1,0.2 \ldots, 1\}$.

\begin{tabular}{|c||c|c|c|c|c|c|c|c||c|c|}
\hline$\sigma$ & $x_{1}^{E \ell}$ & $x_{2}^{E f}$ & $\bar{x}$ & $y_{1}^{E \ell}$ & $y_{2}^{E f}$ & $p_{1}^{M}$ & $p_{1}^{D}$ & $p_{2}^{D}$ & $V_{1}^{E \ell}$ & $V_{2}^{E f}$ \\
\hline \hline 0.0001 & 0.5 & 1 & 0.583 & 0.0336 & 11.5 & 29.8 & 0.58 & 0.42 & 1.67 & 0.156 \\
\hline 0.1 & 0.5 & 1 & 0.583 & 0.0353 & 12.1 & 29.8 & 0.58 & 0.42 & 1.61 & 0.157 \\
\hline 0.2 & 0.5 & 1 & 0.583 & 0.0404 & 13.8 & 29.8 & 0.58 & 0.42 & 1.45 & 0.159 \\
\hline 0.3 & 0.5 & 1 & 0.583 & 0.0488 & 16.7 & 29.8 & 0.58 & 0.42 & 1.23 & 0.161 \\
\hline 0.4 & 0.263 & 1 & 0.548 & 0.0613 & 11.7 & 29.5 & 0.80 & 0.67 & 1.09 & 0.289 \\
\hline 0.5 & 0.055 & 1 & 0.509 & 0.0775 & 9.9 & 29.1 & 0.96 & 0.93 & 0.954 & 0.435 \\
\hline 0.6 & 0 & 1 & 0.5 & 0.0968 & 11.2 & 29 & 1 & 1 & 0.863 & 0.482 \\
\hline 0.7 & 0 & 1 & 0.5 & 0.119 & 13.8 & 29 & 1 & 1 & 0.795 & 0.485 \\
\hline 0.8 & 0 & 1 & 0.5 & 0.145 & 16.8 & 29 & 1 & 1 & 0.743 & 0.487 \\
\hline 0.9 & 0 & 1 & 0.5 & 0.174 & 20.2 & 29 & 1 & 1 & 0.703 & 0.489 \\
\hline 1 & 0 & 1 & 0.5 & 0.207 & 24.0 & 29 & 1 & 1 & 0.671 & 0.491 \\
\hline
\end{tabular}

Table 1: The outcome of the subgame perfect equilibrium depends on the values of $\bar{u}=30$, $c=1, r=0.1, \alpha=0.099, \kappa=2, F=10$, and $y=0.001$. The last two columns, $V_{1}^{E \ell}$ and $V_{2}^{E f}$, represent the values of the leader and the follower at the subgame perfect equilibrium.

First, we briefly discuss how the volatility $\sigma$ influences the thresholds $x_{1}^{E \ell}$ and $x_{2}^{E f}$. Table 1 shows that the locations of the two firms $x_{1}^{E \ell}$ is decreasing in $\sigma$, whereas $x_{2}^{E f}=1$ always holds. Thus, $\sigma$ affects only the equilibrium location of firm 1. Numerical calculations confirm the validity of Lemma 2 and Proposition 1 . 
Second, we discuss the relationship between $y_{1}^{E \ell}$ or $y_{2}^{E f}$ and $\sigma$. To investigate the effect of $\sigma$, the following decomposition is useful:

$$
\frac{\mathrm{d} y_{i}^{E}}{\mathrm{~d} \sigma}=\frac{d y_{i}^{E}\left(\beta(\sigma), x_{1}^{E \ell}(\sigma)\right)}{\mathrm{d} \sigma}=\frac{\partial y_{i}^{E}}{\partial \beta} \frac{\partial \beta}{\partial \sigma}+\frac{\partial y_{i}^{E}}{\partial x_{1}} \frac{\partial x_{1}^{E \ell}}{\partial \sigma}, \quad i=1,2 .
$$

We call the first term of (21) the option effect; this is induced through the parameter $\beta$. The option effect always appears in a standard real options model, and is monotonically increasing in the volatility $\sigma$. The second term of (21), called the location effect, is the effect induced through the leader's location $x_{1}^{E \ell}$. The location effect is new in the literature and should be considered in the model where the strategy of each firm is multidimensional.

First, we examine the effect of $\sigma$ on the threshold of the leader firm, $y_{1}^{E \ell}$. Note again that the first term of (21) is strictly positive. For the second term, we easily verify that $\partial y_{1}^{E \ell} / \partial x_{1}$ and $\partial x_{1}^{E \ell} / \partial \sigma$ are both non-positive. In other words, the option and location effects are both positive and the leader's threshold is always increasing with respect to volatility.

However, the story is different for the effect of $\sigma$ on $y_{2}^{E f}$. This is because although the option effect is still positive for $y_{2}^{E f}$, the location effect, or the effect of $\sigma$ via the leader's location, can be negative, because $\partial y_{2}^{E f} / \partial x_{1}>0$ and $\partial x_{1}^{E \ell} / \partial \sigma$ is strictly negative when $x_{1}^{E \ell}$ is on $(0,1 / 2)$. In other words, the option effect is positive but the location effect can be negative for the follower's threshold. Further, the total effect (21) is negative if the location effect dominates the option effect. Our numerical result shows that this can happen with our parameter setting (see $y_{i}^{E f}$ when $\sigma=0.3,0.4,0.5$ in Table 1).

Now, let us explain why $\sigma$ has a negative effect on $y_{2}^{E f}$ if it takes an intermediate value. If $x_{1}^{E \ell}=0$ or $x_{1}^{E \ell}=1 / 2$, the follower knows that the leader would not change its location $x_{1}^{E \ell}$ for a marginal change in $\sigma$ and thus considers only the option effect, leading to $\sigma$ having a positive effect on $y_{2}^{E f}$. On the other hand, if $x_{1}^{E \ell}$ is between 0 and $1 / 2$, the follower actually takes the marginal change of $x_{1}^{E \ell}$ that affects the present value of its own future profit flows. Because the leader chooses a location closer to 0, the present value 
of the follower becomes higher and the follower can enter the market more easily. Hence, the follower optimally chooses a lower $y_{2}^{E f}$.

Mason and Weeds (2010) show that the threshold of a leader is non-monotonic if there is a positive externality. However, to the authors' best knowledge, the negative effect of the volatility on a threshold for some parameter settings is new in the real options literature under the assumption of a negative externality. It should be mentioned that Mason and Weeds (2010) investigate all three types of equilibria and that non-monotonicity occurs in a preemptive equilibrium. Thus, the mechanism behind our result is quite different from theirs. The result is due to our model setup in which each firm optimizes the threshold and location, confirming the importance of the analysis of multi-dimensional strategies.

\section{Welfare Analysis}

In this section, we conduct welfare analysis of the case where a social planner can sequentially invest twice. There are many ways to assume by which variables the social planner can choose the thresholds, locations, or prices. ${ }^{13}$ In particular, we examine one of the seven cases where the social planner can freely choose its thresholds and locations. This setting is similar to the welfare analysis presented in page 391 of Huisman and Kort (2015).

\footnotetext{
${ }^{13}$ Some examples are presented below.
}

(1) First best, where the social planner can choose all the strategic variables - the entry timings, locations, and prices.

(2-1) Second best, where the social planner can choose the entry timings and locations, but not the prices.

(2-2) Second best, where the social planner can choose the entry timings and prices, but not the locations.

(2-3) Second best, where the social planner can choose the locations and prices, but not the entry timings.

(3-1) Third best, where the social planner can choose the entry timings, but not the locations or prices.

(3-2) Third best, where the social planner can choose the locations, but not the entry timings or prices.

(3-3) Third best, where the social planner can choose the prices, but not the entry timings or locations. Following Huisman and Kort (2015), we opt for (2-1) from among the seven cases. 
Under the assumption, a price competition occurs between two firms after their entry. Then, the equilibrium prices are the same as in the autonomous economy studied in the previous sections; they are given by $p_{1}^{M}\left(x_{1}\right), p_{1}^{D \ell}\left(x_{1}, x_{2}\right)$, and $p_{2}^{D f}\left(x_{1}, x_{2}\right)$ in (5) and (66) . The social planner sets the thresholds and locations so as to maximize the social surplus defined below.

\subsection{Analytical results}

In the social planner's problem, we refer to firms 1 and 2 as the first and the second entrants, respectively. On the other hand, if we consider the main model of the autonomous economy studied in the previous sections, we refer to the leader and the follower as the first and the second entrants, respectively.

Before the analysis, let us define the consumer and producer surpluses. The total expected consumer surplus is

$$
C S\left(y ; y_{1}, y_{2}, x_{1}, x_{2}\right)=\mathbb{E}_{y}\left[\mathrm{e}^{-r\left(T_{1}\left(y_{1}\right)-t\right)} H_{1}\left(y ; y_{2}, x_{1}, x_{2}\right)\right]
$$

where

$$
H_{1}\left(y ; y_{2}, x_{1}, x_{2}\right)=\mathbb{E}_{y}\left[\int_{t}^{T_{2}\left(y_{2}\right)} c s^{M}\left(x_{1}\right) \mathrm{e}^{-r(s-t)} Y_{s} \mathrm{~d} s+\int_{T_{2}\left(y_{2}\right)}^{\infty} c s^{D}\left(x_{1}, x_{2}\right) \mathrm{e}^{-r(s-t)} Y_{s} \mathrm{~d} s\right],
$$

and

$$
\begin{aligned}
c s^{M}\left(x_{1}\right)= & \int_{0}^{1}\left[\bar{u}-\left(p_{1}^{M}\left(x_{1}\right)+c\left(x-x_{1}\right)^{2}\right)\right] \mathrm{d} x, \\
c s^{D}\left(x_{1}, x_{2}\right)= & \int_{0}^{\bar{x}}\left[\bar{u}-\left(p_{1}^{D \ell}\left(x_{1}, x_{2}\right)+c\left(x-x_{1}\right)^{2}\right)\right] \mathrm{d} x \\
& +\int_{\bar{x}}^{1}\left[\bar{u}-\left(p_{2}^{D f}\left(x_{1}, x_{2}\right)+c\left(x-x_{2}\right)^{2}\right)\right] \mathrm{d} x .
\end{aligned}
$$

The right-hand side of (22) can be easily calculated as

$$
C S\left(y ; y_{1}, y_{2}, x_{1}, x_{2}\right)=\left(\frac{y_{1} c s^{M}\left(x_{1}\right)}{r-\alpha}\right)\left(\frac{y}{y_{1}}\right)^{\beta}+\left(\frac{y_{2}\left[c s^{D}\left(x_{1}, x_{2}\right)-c s^{M}\left(x_{1}\right)\right]}{r-\alpha}\right)\left(\frac{y}{y_{2}}\right)^{\beta} \text {. }
$$


On the other hand, the total expected producer surplus is equal to the sum of the values of the two firms; that is,

$$
P S\left(y ; y_{1}, y_{2}, x_{1}, x_{2}\right)=V_{1}\left(y ; y_{1}, y_{2}, x_{1}, x_{2}\right)+V_{2}\left(y ; y_{1}, y_{2}, x_{1}, x_{2}\right)
$$

Then, the total expected surplus is naturally defined by the sum of the consumer and producer surpluses as

$$
T S\left(y ; y_{1}, y_{2}, x_{1}, x_{2}\right)=C S\left(y ; y_{1}, y_{2}, x_{1}, x_{2}\right)+P S\left(y ; y_{1}, y_{2}, x_{1}, x_{2}\right)
$$

We can easily calculate that

$$
\begin{aligned}
& T S\left(y ; y_{1}, y_{2}, x_{1}, x_{2}, p_{1}, p_{2}\right) \\
= & \left(\frac{y_{1} t s^{M}\left(x_{1}\right)}{r-\alpha}-F_{1}\right)\left(\frac{y}{y_{1}}\right)^{\beta}+\left(\frac{y_{2}\left[t s^{D}\left(x_{1}, x_{2}\right)-t s^{M}\left(x_{1}\right)\right]}{r-\alpha}-F_{2}\right)\left(\frac{y}{y_{2}}\right)^{\beta},
\end{aligned}
$$

where

$$
\begin{aligned}
t s^{M}\left(x_{1}\right) & =\int_{0}^{1}\left[\bar{u}-c\left(x-x_{1}\right)^{2}\right] \mathrm{d} x \\
t s^{D}\left(x_{1}, x_{2}\right) & =\int_{0}^{\bar{x}}\left[\bar{u}-c\left(x-x_{1}\right)^{2}\right] \mathrm{d} x+\int_{\bar{x}}^{1}\left[\bar{u}-c\left(x-x_{2}\right)^{2}\right] \mathrm{d} x .
\end{aligned}
$$

The social planner chooses $\left(y ; y_{1}, y_{2}, x_{1}, x_{2}\right)$ such that $T S$ is maximized. By solving the social planner's maximization problem, we have the following proposition.

Proposition 3. The optimal thresholds and locations of the social planner $\left(y_{1}^{O}, y_{2}^{O}, x_{1}^{O}, x_{2}^{O}\right)$ satisfy

$$
\begin{aligned}
x_{1}^{O} & \in(0,1 / 2), \\
x_{2}^{O}\left(x_{1}^{O}\right) & =\frac{28-5 x_{1}-2 \sqrt{76-70 x_{1}^{O}+25\left(x_{1}^{O}\right)^{2}}}{15}, \\
y_{1}^{O}\left(x_{1}^{O}\right) & =\frac{\beta}{\beta-1} \frac{(r-\alpha) F}{t s^{M}\left(x_{1}^{O}\right)} \\
y_{2}^{O}\left(x_{1}^{O}\right) & =\frac{\beta}{\beta-1} \frac{(r-\alpha) \kappa F}{\left[t s^{D}\left(x_{1}^{O}, x_{2}^{O}\right)-t s^{M}\left(x_{1}^{O}\right)\right]} .
\end{aligned}
$$


Proof. See Appendix A.

From Proposition 3, we characterize the two locations and thresholds in our equilibrium solution by comparing the outcomes in the social planner's problem. For example, the two locations chosen by the social planner are always interior $\left(x_{1}^{O} \in(0,1 / 2)\right.$, $\left.x_{2}^{O} \in(4(7-\sqrt{19}) / 15,(17-2 \sqrt{21}) / 10)\right)$, whereas at least the follower's location is at the edge at the $\operatorname{SPNE}\left(x_{2}^{E}=1\right)$. From a consumers' viewpoint, the equilibrium outcome in the main model is worse than that in the social planner's problem, because consumers need to pay more transportation costs. A welfare loss apparently occurs in our main model without a social planner.

As for the thresholds of the social optimum and the SPNE, we obtain the following corollary:

Corollary 2. (i) $y_{1}^{O} \gtreqless y_{1}^{E \ell}$ if and only if $x_{1}^{E \ell}+\left(x_{1}^{E \ell}-x_{1}^{O}\right)\left[1-\left(x_{1}^{E \ell}+x_{1}^{O}\right)\right] \gtreqless 2 / 3$. Furthermore, $y_{1}^{O}<y_{1}^{E \ell}$ if $x_{1}^{O} \in[1 / 4,1 / 2]$ or $x_{1}^{O} \geq x_{1}^{E \ell}$.

(ii) $y_{2}^{O} \gtreqless y_{2}^{E f}$ if and only if $2\left(x_{2}^{E f}-x_{1}^{E \ell}\right)\left(4-x_{1}^{E \ell}-x_{2}^{E f}\right)^{2} \gtreqless\left(x_{2}^{O}-x_{1}^{O}\right)\left(4-x_{1}^{O}-x_{2}^{O}\right)(8-$ $\left.5 x_{1}^{O}-x_{2}^{O}\right)$. Furthermore, if $x_{1}^{O}>0.162$, then $y_{2}^{O}>y_{2}^{E f}$.

Proof. See Appendix B.

Next we discuss Corollary 2 in more detail. Note that for the threshold of the leader, the social planner sets firm 1 in the market earlier than the leader in the main model $\left(y_{1}^{O}<y_{1}^{E}\right)$ for a fixed $x_{1}$ because $t s^{M}\left(x_{1}\right)>\pi_{1}^{M \ell}\left(x_{1}\right)$. Inequality $t s^{M}\left(x_{1}\right)>\pi_{1}^{M \ell}\left(x_{1}\right)$ still holds when $x_{1}^{O} \geq x_{1}^{E \ell}$ and the difference between $x_{1}^{O}$ and $x_{1}^{E \ell}$ is small. Then, the social planner lets firm 1 enter earlier because the present value of the future profit is higher than in the main model. Corollary 2 (i) confirms this observation.

For the threshold of the follower, the social planner should avoid price competition between the two firms for a low value of $y$. Consequently, the time interval between the two entries chosen by the firms in the main model is shorter than that chosen by the 
social planner, especially if $x_{1}^{O}$ is closer to $1 / 2$. Corollary 2 (ii) can be justified by the above explanation.

\subsection{Numerical results}

Finally, we conduct a numerical analysis of the results of the corollaries. As in Table 1, we discuss how $\sigma$ affects the strategic variables and levels of the social surplus. Table 2 shows the outcomes of the strategic variables and levels of the social surplus. Suppose that the parameter values set are the same as in Table 1 , $\bar{u}=30, c=1, r=0.1, \alpha=0.99$, $\kappa=2, F=10$, and $y=0.001$.

\begin{tabular}{|c||c|c|c|c|c|c|c|c||c|c|}
\hline$\sigma$ & $x_{1}^{O}$ & $x_{2}^{O}$ & $\bar{x}^{O}$ & $y_{1}^{O}$ & $y_{2}^{O}$ & $p_{1}^{M}$ & $p_{1}^{D}$ & $p_{2}^{D}$ & $W^{O}$ & $W^{E}$ \\
\hline \hline 0.0001 & 0.430 & 0.776 & 0.534 & 0.0334 & 38 & 29.68 & 0.37 & 0.32 & 28.63 & 28.60 \\
\hline 0.1 & 0.425 & 0.775 & 0.533 & 0.0351 & 39 & 29.67 & 0.37 & 0.33 & 28.68 & 28.65 \\
\hline 0.2 & 0.413 & 0.774 & 0.531 & 0.0402 & 41 & 29.66 & 0.38 & 0.34 & 28.81 & 28.78 \\
\hline 0.3 & 0.396 & 0.771 & 0.528 & 0.0486 & 46 & 29.64 & 0.40 & 0.35 & 28.97 & 28.94 \\
\hline 0.4 & 0.378 & 0.769 & 0.525 & 0.0603 & 51 & 29.61 & 0.41 & 0.37 & 29.13 & 29.11 \\
\hline 0.5 & 0.360 & 0.767 & 0.521 & 0.0754 & 59 & 29.59 & 0.42 & 0.39 & 29.27 & 29.22 \\
\hline 0.6 & 0.345 & 0.764 & 0.518 & 0.0939 & 68 & 29.57 & 0.44 & 0.40 & 29.39 & 29.32 \\
\hline 0.7 & 0.331 & 0.762 & 0.516 & 0.116 & 78 & 29.55 & 0.44 & 0.42 & 29.48 & 29.42 \\
\hline 0.8 & 0.319 & 0.761 & 0.513 & 0.141 & 90 & 29.54 & 0.45 & 0.43 & 29.55 & 29.49 \\
\hline 0.9 & 0.310 & 0.759 & 0.512 & 0.169 & 104 & 29.52 & 0.46 & 0.44 & 29.61 & 29.55 \\
\hline 1 & 0.302 & 0.758 & 0.510 & 0.201 & 119 & 29.51 & 0.47 & 0.45 & 29.66 & 29.60 \\
\hline
\end{tabular}

Table 2: The outcomes of the strategic variables when the social planners choose both the thresholds and locations depending on the values of $\bar{u}=30, c=1, r=0.1, \alpha=0.099$, $\kappa=2, F=10$, and $y=0.001$. The last two columns represent the level of social surplus at the social optimum and SPNE.

The major finding from Table 2 is that $W^{O}-W^{E}$ is increasing in $\sigma$. In other words, a welfare loss in the main model becomes larger as the volatility gets higher. This finding can be explained by comparing $x_{1}^{E \ell}$ and $x_{1}^{O}$. Note that $x_{1}^{O}>1 / 4$, and that for a large $\sigma$, the leader firm optimally choose $x_{1}^{E \ell}=0$. Then, the difference between $x_{1}^{E \ell}$ and $x_{1}^{O}$ is quite significant and the price competition is less severe in the main model, and this 
leads to a large welfare loss. Note that this finding is obtained only in our model with multi-dimensional strategies. The novelty of the current study is confirmed by welfare analysis.

\section{Conclusion}

In this article, we construct a Hotelling-type spatial model with uncertainty of market demand in a real options framework. Contrary to the standard real options literature, the strategy of each firm is multi-dimensional in that each firm maximizes its value function with respect to multiple variables, entry timing, and location. This multi-dimensional approach leads to many interesting results. One important finding is that the threshold of the follower is not monotonically increasing in volatility, which is new in the literature, and can be obtained only with multi-dimensional strategies. Also, although the follower's entry timing tends to be late as the volatility increases, the leader is more likely to locate at the edge as the volatility gets higher. The observations on the locations and welfare analysis also shed new light on the real options and IO literatures.

The most important point for a future study is the preemptive equilibrium in our model. Other future studies need to consider a circular city model and generalize the stochastic process $Y$, for example. However, as a final remark, we argue that the results obtained in this study are not restrictive and can be applied to many actual situations in the IO problems.

\section{A Proof of Proposition 3}

First, let us consider the second-entry problem of the social planner, $y_{2}$ and $x_{2}$. To solve the second-entry problem, we differentiate (23) with respect to $x_{2}$, to have

$$
x_{2}^{O}\left(x_{1}\right)=\frac{28-5 x_{1}-2 \sqrt{76-70 x_{1}+25 x_{1}^{2}}}{15} .
$$


Next, by differentiating (23) with respect to $y_{2}$, we have

$$
y_{2}^{O}\left(x_{1}\right)=\frac{\beta}{\beta-1} \frac{(r-\alpha) F_{2}}{\left[t s^{D}\left(x_{1}, x_{2}^{O}\left(x_{1}\right)\right)-t s^{M}\left(x_{1}\right)\right]} .
$$

Second, as with the second entry of the social planner, we consider the maximization problem of the first entry. By differentiating Equation (23) with respect to $y_{1}$, we obtain the optimal threshold of the first entry as follows:

$$
y_{1}^{O}\left(x_{1}\right)=\frac{\beta}{\beta-1} \frac{(r-\alpha) F_{1}}{t s^{M}\left(x_{1}\right)} .
$$

To obtain the optimal location of the first entry, we differentiate (23) with respect to $x_{1}$ and substitute the above optimal reaction functions with respect to $x_{1}, x_{2}^{O}\left(x_{1}\right), y_{1}^{O}\left(x_{1}\right)$, and $y_{2}^{O}\left(x_{1}\right)$, into the first-order derivative. Then, we have

$$
\begin{aligned}
& \left.\frac{\partial T S\left(y ; y_{1}, y_{2}, x_{1}, x_{2}\right)}{\partial x_{1}}\right|_{y_{1}=y_{1}^{O}\left(x_{1}\right), y_{2}=y_{2}^{O}\left(x_{1}\right), x_{2}=x_{2}^{O}\left(x_{1}\right)} \\
= & \frac{y_{1}^{O}\left(x_{1}\right) t s^{M \prime}\left(x_{1}\right)}{r-\alpha}\left(\frac{y}{y_{1}^{O}\left(x_{1}\right)}\right)^{\beta}+\frac{y_{2}^{O}\left(x_{1}\right)\left[\partial t s^{D}\left(x_{1}, x_{2}\right) /\left.\partial x_{1}\right|_{x_{2}=x_{2}^{O}\left(x_{1}\right)}-t s^{M \prime}\left(x_{1}\right)\right]}{r-\alpha}\left(\frac{y}{y_{2}^{O}\left(x_{1}\right)}\right)^{\beta} .
\end{aligned}
$$

Let the right-hand side of (24) denote $Z_{1}$. The optimal location of the social planner is interior if (24) becomes 0 for some $x_{1} \in(0,1.2)$. It can be calculated that

$$
\begin{aligned}
\lim _{x_{1} \rightarrow 0} Z_{1} & =\frac{3 F}{4} \frac{\beta}{\beta-1}\left[\frac{\beta-1}{\beta(r-\alpha) F}\right]^{\beta}\left\{4 c\left(\bar{u}-\frac{c}{3}\right)^{\beta-1}-\frac{2^{5 \beta}}{3^{5 \beta} 5^{2 \beta-1}} \frac{11+7 \sqrt{19}}{(19 \sqrt{19}-28)^{\beta-1}} \frac{c^{\beta}}{\kappa^{\beta-1}}\right\} \\
& >\frac{3 F}{4} \frac{\beta}{\beta-1}\left[\frac{\beta-1}{\beta(r-\alpha) F}\right]^{\beta}\left\{4 c\left(3 c-\frac{c}{3}\right)^{\beta-1}-\frac{2^{5 \beta}}{3^{5 \beta} 5^{2 \beta-1}} \frac{11+7 \sqrt{19}}{(19 \sqrt{19}-28)^{\beta-1}} \frac{c^{\beta}}{1^{\beta-1}}\right\} \\
& =\frac{3 c^{\beta} F}{4} \frac{\beta}{\beta-1}\left[\frac{\beta-1}{\beta(r-\alpha) F}\right]^{\beta}\left\{4\left(\frac{8}{3}\right)^{\beta-1}-\frac{2^{5 \beta}}{3^{5 \beta} 5^{2 \beta-1}} \frac{11+7 \sqrt{19}}{(19 \sqrt{19}-28)^{\beta-1}}\right\}>0
\end{aligned}
$$

and

$$
\lim _{x_{1} \rightarrow 1 / 2} Z_{1}=-\frac{\beta}{\beta-1} \frac{2^{\beta+1}(6+\sqrt{21}) \kappa F}{3(27+7 \sqrt{21})^{\beta}}\left(\frac{(\beta-1) c}{\beta(r-\alpha) \kappa F}\right)^{\beta}<0 .
$$

Thus, because $Z_{1}$ is continuous in $x_{1}$, the optimal location of the first entry for the social planner becomes interior. That is, $x_{1}^{O} \in(0,1 / 2)$. 


\section{B Proof of Corollary 2}

(i) We simply calculate the subtraction of $y_{1}^{E l l}$ and $y_{1}^{O}$ as

$$
\begin{aligned}
y_{1}^{O}-y_{1}^{E \ell} & =\frac{\beta}{\beta-1} \frac{(r-\alpha) F}{t s^{M}\left(x_{1}^{O}\right)}-\frac{\beta}{\beta-1} \frac{(r-\alpha) F}{\pi_{1}^{M \ell}\left(x_{1}^{E \ell}\right)} \\
& =\frac{\beta}{\beta-1} \frac{(r-\alpha) F}{t s^{M}\left(x_{1}^{O}\right) \pi_{1}^{M \ell}\left(x_{1}^{E \ell}\right)}\left(\pi_{1}^{M \ell}\left(x_{1}^{E \ell}\right)-t s^{M}\left(x_{1}^{O}\right)\right) \\
& =\frac{\beta}{\beta-1} \frac{(r-\alpha) F}{t s^{M}\left(x_{1}^{O}\right) \pi_{1}^{M \ell}\left(x_{1}^{E \ell}\right)} c\left(-\frac{2}{3}+2 x_{1}^{E \ell}-\left(x_{1}^{E \ell}\right)^{2}+\left(x_{1}^{O}\right)^{2}-x_{1}^{O}\right) \\
& =\frac{\beta}{\beta-1} \frac{(r-\alpha) F}{t s^{M}\left(x_{1}^{O}\right) \pi_{1}^{M \ell}\left(x_{1}^{E \ell}\right)} c\left(\frac{1}{12}-\left(1-x_{1}^{E \ell}\right)^{2}+\left(\frac{1}{2}-x_{1}^{O}\right)^{2}\right) \\
& =\frac{\beta}{\beta-1} \frac{(r-\alpha) F}{t s^{M}\left(x_{1}^{O}\right) \pi_{1}^{M \ell}\left(x_{1}^{E \ell}\right)} c\left(-\frac{2}{3}+x_{1}^{E \ell}+\left(x_{1}^{E \ell}-x_{1}^{O}\right)\left[1-\left(x_{1}^{E \ell}+x_{1}^{O}\right)\right]\right) .
\end{aligned}
$$

(ii) Similarly, we calculate the subtraction as

$$
\begin{aligned}
y_{2}^{O}-y_{2}^{E \ell}= & \frac{\beta}{\beta-1} \frac{(r-\alpha) F}{\left[t s^{D}\left(x_{1}^{O}, x_{2}^{O}\right)-t s^{M}\left(x_{1}^{O}\right)\right] \pi_{2}^{D f}\left(x_{1}^{E \ell}\right)}\left(\pi_{2}^{D f}\left(x_{1}^{E \ell}\right)+t s^{M}\left(x_{1}^{O}\right)-t s^{D}\left(x_{1}^{O}, x_{2}^{O}\right)\right) \\
= & \frac{\beta}{\beta-1} \frac{(r-\alpha) F}{\left[t s^{D}\left(x_{1}^{O}, x_{2}^{O}\right)-t s^{M}\left(x_{1}^{O}\right)\right] \pi_{2}^{D f}\left(x_{1}^{E \ell}\right)} \frac{c}{36} \\
& \times\left\{2\left(x_{2}^{E f}-x_{1}^{E \ell}\right)\left(4-x_{1}^{E \ell}-x_{2}^{E f}\right)^{2}-\left(x_{2}^{O}-x_{1}^{O}\right)\left(4-x_{1}^{O}-x_{2}^{O}\right)\left(8-5 x_{1}^{O}-x_{2}^{O}\right)\right\} .
\end{aligned}
$$

The first and second parts of the square brackets are denoted as $X^{E} \equiv 2\left(x_{2}^{E f}-x_{1}^{E \ell}\right)(4-$ $\left.x_{1}^{E \ell}-x_{2}^{E f}\right)^{2}$ and $X^{O} \equiv\left(x_{2}^{O}-x_{1}^{O}\right)\left(4-x_{1}^{O}-x_{2}^{O}\right)\left(8-5 x_{1}^{O}-x_{2}^{O}\right)$, respectively. Note that both $X^{E}$ and $X^{O}$ are decreasing in $x_{1}^{E} \in[0,1 / 2]$ and $x_{1}^{O} \in(0,1 / 2)$. Thus, $X^{E}$ is minimized at $x_{1}^{E \ell}=1 / 2$ and equals 25/4. By solving $X^{O}=25 / 4$, we have $x_{1}^{O}=0.1619 \cdots$. Therefore, if $x_{1}^{O}>0.162, X^{E}>X^{O}$ holds for any $x_{1}^{E \ell} \in[0,1 / 2]$. Thus, we have the desired results.

\section{References}

Bonanno, G. (1987), "Location Choice, Product Proliferation and Entry Deterrence," Review of Economic Studies, 54(1), 37-45. 
Bronnenberg, B. J. and V. Mahajan (2001), "Unobserved Retailer Behavior in Multimarket Data: Joint Spatial Dependence in Market Shares and Promotion Variables," Marketing Science, 20(3), 284-299.

Chevalier-Roignant, B., C. M. Flath, A. Huchzermeier, and L. Trigeorgis (2011), "Strategic Investment under Uncertainty: A Synthesis," European Journal of Operational Research, 215(3), 639-650.

Christou, C. and N. Vettas (2005), "Location Choices under Quality Uncertainty," Mathematical Social Sciences, 50(3), 268-278.

Cleeren, K., F. Verboven, M. G. Dekimpe, and K. Gielens (2010), "Intra- and Interformat Competition among Discounters and Supermarkets," Marketing Science, 29(3), $456-473$.

d'Aspremont, C., J. J. Gabszewicz, and J.-F. Thisse (1979), "On Hotelling's 'Stability in Competition'," Econometrica, 47(5), 1145-1150.

Ebina, T., N. Matsushima, and D. Shimizu (2015), "Product Differentiation and Entry Timing in a Continuous-Time Spatial Competition Model," European Journal of Operational Research, 247(3), 904-913.

Friedman, J.W. and J.-F. Thisse (1993), "Partial Collusion Fosters Minimum Product Differentiation," RAND Journal of Economics, 24(4), 631-645.

Grenadier, S. R. (1996), "The Strategic Exercise of Options: Development Cascades and Overbuilding in Real Estate Markets," Journal of Finance, 51(5), 1653-1679.

Hotelling, H. (1929), "Stability in Competition," Economic Journal, 39(153), 41-57.

Huisman, K. J. M. and P. M. Kort (2015), "Strategic Investment in Technological Innovations," European Journal of Operational Research, 144(1), 209-223. 
Huisman, K. J. M. and P. M. Kort (2015), "Strategic Capacity Investment under Uncertainty," RAND Journal of Economics, 46(2), 376-408.

Hwang, M., B. J. Bronnenberg, and R. Thomadsen (2010), "An Empirical Analysis of Assortment Similarities across U.S. Supermarkets," Marketing Science, 29(5), 858-879.

Kim, H. and K. Serfes (2006), "A Location Model with Preference for Variety," Journal of Industrial Economics, 54(4), 569-595.

Lages, L. F. and J. C. Fernandes (2005), "The SERPVAL Scale: A Multi-Item Instrument for Measuring Service Personal Values," Journal of Business Research, 58(11), 1562 1572 .

Lai, L. F. and T. Tabuchi (2012), "Hotelling Meets Weber," Regional Science and Urban Economics, 42(6), 1017-1022.

Lambertini, L. (2002), "Equilibrium Locations in a Spatial Model with Sequential Entry in Real Time," Regional Science and Urban Economics, 32(1), 47-58.

Loertscher, S. and G. Muehlheusser (2011), "Sequential Location Games," RAND Journal of Economics, 42(4), 639-663.

Mason, R. and H. Weeds (2010), "Investment, Uncertainty and Pre-emption," International Journal of Industrial Organization, 28(3), 278-287.

Matsushima, N. (2009), "Vertical Mergers and Product Differentiation," Journal of Industrial Economics, 57(4), 812-834.

Meagher, K. J. and K. G. Zauner (2004), "Product Differentiation and Location Decisions under Demand Uncertainty," Journal of Economic Theory, 117(2), 201-216.

Neven, D. J. (1987), "Endogenous Sequential Entry in a Spatial Model," International Journal of Industrial Organization, 5(4), 419-434. 
Nielsen, M. J. (2002), "Competition and Irreversible Investments," International Journal of Industrial Organization, 20(5), 731-743.

Pawlina, G. and P. M. Kort (2006), "Real Options in an Asymmetric Duopoly: Who Benefits from Your Competitive Disadvantage?" Journal of Economics and Management Strategy, 15(1), 1-35.

Tabuchi, T. and J.-F. Thisse (1995), "Asymmetric Equilibria in Spatial Competition," International Journal of Industrial Organization, 13(2), 213-227.

Thomadsen, R. (2007), "Product Positioning and Competition: The Role of Location in the Fast Food Industry," Marketing Science, 26(6), 792-804.

Vakratsas, D. and C. Kolsarici (2008), "A Dual-Market Diffusion Model for a New Prescription Pharmaceutical," International Journal of Research in Marketing, 25(4), 282293.

Victor, N. M. and J. H. Ausubel (2002), "DRAMs as Model Organisms for Study of Technological Evolution," Technological Forecasting and Social Change, 69(3), 243262.

Weeds H. (2002), "Strategic Delay in a Real Options Model of R\&D Competition," Review of Economic Studies, 69(3), 729-747. 